\title{
Probing the Mechanical Strength of an Armored Bubble and Its Implication to Particle-Stabilized Foams
}

\author{
Nicolas Taccoen, ${ }^{1}$ François Lequeux, ${ }^{2}$ Deniz Z. Gunes, ${ }^{3}$ and Charles N. Baroud ${ }^{1, *}$ \\ ${ }^{1}$ LadHyX and Department of Mechanics, Ecole Polytechnique, CNRS, 91128 Palaiseau Cedex, France \\ ${ }^{2}$ École Supérieure de Physique et de Chimie Industrielles de la Ville de Paris (ESPCI) ParisTech, \\ PSL Research University, Sciences et Ingénierie de la matière Molle, CNRS UMR7615, \\ 10, Rue Vauquelin, F-75231 Paris Cedex 05, France \\ ${ }^{3}$ Food Science and Technology Department, Nestle Research Center, Vers-Chez-Les-Blanc, \\ CH-1000 Lausanne 26, Switzerland
}

(Received 3 July 2015; revised manuscript received 22 December 2015; published 5 February 2016)

Bubbles are dynamic objects that grow and rise or shrink and disappear, often on the scale of seconds. This conflicts with their uses in foams where they serve to modify the properties of the material in which they are embedded. Coating the bubble surface with solid particles has been demonstrated to strongly enhance the foam stability, although the mechanisms for such stabilization remain mysterious. In this paper, we reduce the problem of foam stability to the study of the behavior of a single spherical bubble coated with a monolayer of solid particles. The behavior of this armored bubble is monitored while the ambient pressure around it is varied, in order to simulate the dissolution stress resulting from the surrounding foam. We find that above a critical stress, localized dislocations appear on the armor and lead to a global loss of the mechanical stability. Once these dislocations appear, the armor is unable to prevent the dissolution of the gas into the surrounding liquid, which translates into a continued reduction of the bubble volume, even for a fixed overpressure. The observed route to the armor failure therefore begins from localized dislocations that lead to large-scale deformations of the shell until the bubble completely dissolves. The critical value of the ambient pressure that leads to the failure depends on the bubble radius, with a scaling of $\Delta P_{\text {collapse }} \propto R^{-1}$, but does not depend on the particle diameter. These results disagree with the generally used elastic models to describe particle-covered interfaces. Instead, the experimental measurements are accounted for by an original theoretical description that equilibrates the energy gained from the gas dissolution with the capillary energy cost of displacing the individual particles. The model recovers the short-wavelength instability, the scaling of the collapse pressure with bubble radius, and the insensitivity to particle diameter. Finally, we use this new microscopic understanding to predict the aging of particlestabilized foams, by applying classical Ostwald ripening models. We find that the smallest armored bubbles should fail, as the dissolution stress on these bubbles increases more rapidly than the armor strength. Both the experimental and theoretical results can readily be generalized to more complex particle interactions and shell structures.

DOI: 10.1103/PhysRevX.6.011010

\section{INTRODUCTION}

Foams are ubiquitous and often serve to modify the mechanical [1], acoustical [2], or optical [3] qualities of the objects in which they are embedded. The stability of a foam against aging is therefore a major issue since it determines the ability to control the material properties and their evolution in time. Foams suffer from three major aging processes [4]: First, the density contrast between the gas and liquid leads to liquid drainage towards the bottom of the foam, while the bubbles rise to the top and the films that

\footnotetext{
*baroud@ladhyx.polytechnique.fr
}

Published by the American Physical Society under the terms of the Creative Commons Attribution 3.0 License. Further distribution of this work must maintain attribution to the author(s) and the published article's title, journal citation, and DOI.
Subject Areas: Soft Matter

separate them become thinner. Second, bubbles can merge together, which produces larger bubbles that rise even faster. Finally, gas can diffuse through the liquid from the small to the large bubbles, leading to foam coarsening through a process known as Ostwald ripening.

Surfactants are very efficient at preventing bubble fusions but only have a weak effect on preventing Ostwald ripening. An alternative approach, which has received great interest in recent years, is to coat the air-liquid interfaces of the bubbles with solid particles that prevent bubble fusions, modify their effective density, and also prevent Ostwald ripening [5]. Although these so-called Ramsden foams [6] do display improved stability compared with the pure liquid case (e.g., Refs. [7-11]), the mechanical equilibria that lead to the stabilization remain unclear.

One way to understand the underlying mechanisms is to focus on the behavior of a single particle-covered liquid-air 
interface. However, the difficulty of making quantitative measurements on a single coated bubble implies that most of the work was done by using $\mathrm{mm}$-scale liquid droplets, as surrogate systems for air bubbles [12-18], and aspirating these droplets or letting them evaporate while observing a range of behaviors, depending on the experimental setup. In contrast, experiments using air bubbles, which have shown the arrest of gas dissolution in the liquid, remain mostly qualitative $[19,20]$. Indeed, in spite of the large body of literature on armored drops and bubbles, there are still many open questions on the underlying physical equilibria. For instance, there are no predictions for the stability of these armors as a function of parameters such as bubble and particle sizes, or the thermodynamic environment (pressure, temperature). Moreover, the failure modes of the shells remain unclear, as does the net effect of armoring bubbles within a foam.

Here, we make quantitative measurements of the stability and buckling of a single spherical air bubble, as it dissolves into the surrounding liquid. For this, we use our recently developed microfluidic tools that rely on confinement gradients to produce a single bubble into a quiescent fluid and hold it stationary for observation [21,22]. Such devices have previously been used to observe the flows on bubble surfaces [23], to measure chemical kinetics [24], or to explore the rigidity of giant unilamellar lipid vesicles [25]. Here, the interaction between a bubble and the surrounding foam is simulated by controlling the ambient pressure in the liquid, which allows us to impose a controlled dissolution stress on the bubble. We begin by describing the experimental procedure and results; then a theoretical model of the shell mechanics completes the description. We end by discussing the implications for particle-stabilized foams.

\section{EXPERIMENTS}

\section{A. Bubble generation and coating}

The microfluidic channel and the main steps of bubble production and particle coverage are shown in Fig. 1 (see also movie 1 in Ref. [26]). The device consists of a succession of microfluidic elements: An air inlet leads to a rectangular chamber with a sloping ceiling. Two additional inlets to the chamber serve to bring the particle dispersion and push the whole mix through the coating channel, which then ends in an observation chamber having a conical ceiling. Finally, two channels connect this chamber to the outlets of a pressure controller. The microfabrication details are discussed in Appendix A.

A typical experiment (see movie 1 in Ref. [26]) begins by producing a single air bubble through the injector [Fig. 1(b)], by relying on the confinement gradient in this chamber to produce the bubble into the stationary aqueous phase [22]. Once the bubble is formed, it is pushed through the coating channel by injecting the particle dispersion, which thus progressively coats the bubble with particles

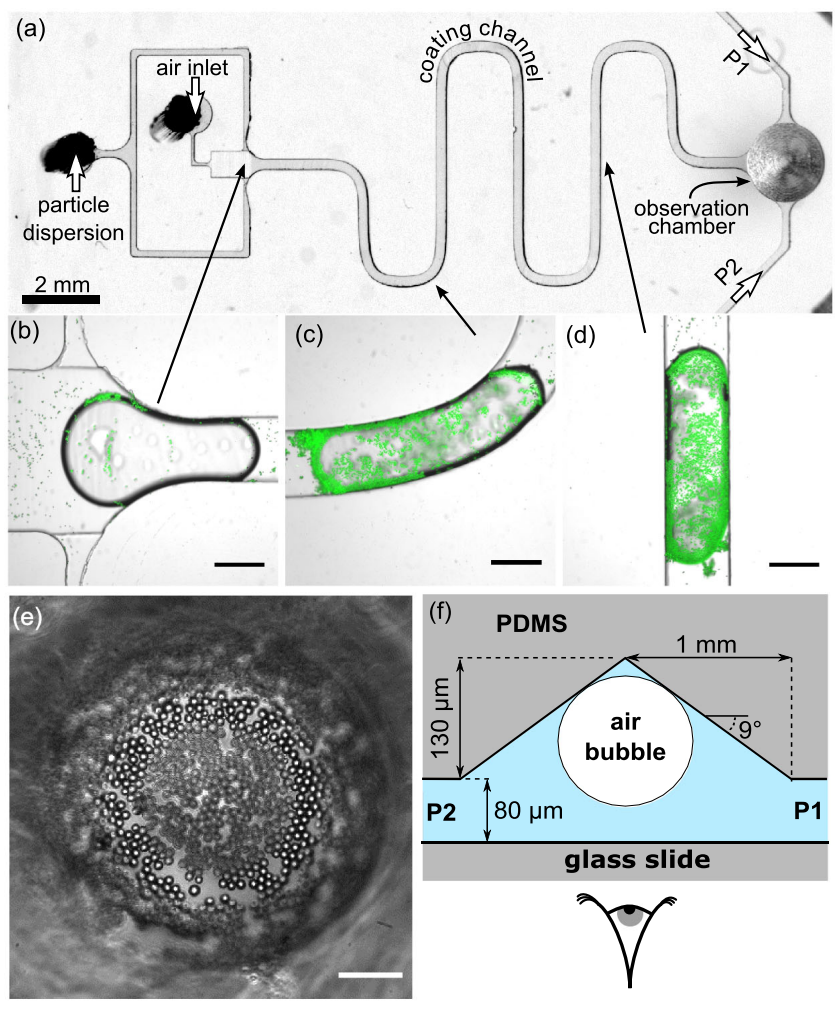

FIG. 1. (a) General view of the microfluidic device which consists of a bubble formation zone, a coating channel, and an observation chamber. (b)-(d) Composite bright-field or fluorescence images where the particles appear in green. (b) First, a slopped injector (height $=30 \mu \mathrm{m}$ to $80 \mu \mathrm{m}$, angle $2.3^{\circ}$ ) produces a single air bubble. (c,d) The bubble is then squeezed through the coating channel by the flow of a particle dispersion where it progressively collects particles. Scale bars are $200 \mu \mathrm{m}$. The armored bubble is then released in the observation chamber, and the excess of unattached particles in suspension is washed away, yielding a bubble coated with a single layer of particles with loosely packed crystalline domains. (e) Phase contrast micrograph of such a bubble with $4.5-\mu \mathrm{m}$ diameter particles. The scale bar is $30 \mu \mathrm{m}$. (f) Cross-section schematic of the conical observation chamber (not to scale). The air bubble is trapped even if the liquid around it is flowed.

[Figs. 1(c) and 1(d)] [27,28]. The coated bubble is then released into the observation chamber [Fig. 1(e)], where it is trapped by the conical ceiling [Fig. 1(f)]. Finally, clean water from a side channel (P1 or P2) is flowed around it to remove the excess of unattached particles. This protocol leaves a single trapped spherical bubble whose interface is armored with a monolayer of loosely packed adsorbed colloids. During the observation, a weak flow ( $v \approx 2 \mu \mathrm{m} / \mathrm{s}$ ) is maintained around the bubble in order to ensure a constant gas concentration in the water.

\section{B. Control of bubble dissolution}

The fate of this bubble depends on the values of the thermodynamic variables in the observation chamber: For a bubble of radius $R$ and at fixed temperature, there exists an 


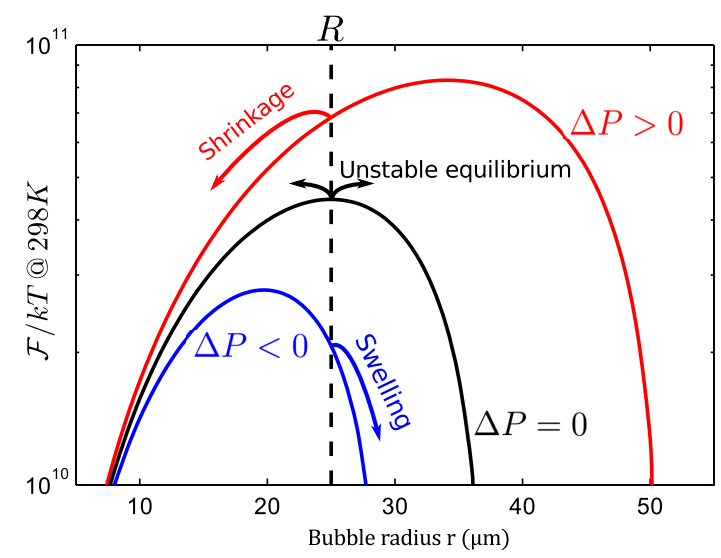

FIG. 2. Free energy of a single gas bubble of radius $R$ in a weak gas-liquid solution. If $\Delta P=0$, the bubble radius is stationary. The bubble shrinks if $\Delta P>0$ and it swells if $\Delta P<0$ because of gas exchange between the liquid and the bubble.

equilibrium value of the ambient pressure $P_{\text {eq }}$ for which the bubble neither shrinks nor grows [29]. This can be seen by writing the free energy of a gas bubble interacting with a gas-liquid solution [30] (see Appendix B for the detailed calculation):

$$
\mathcal{F}=4 \pi \gamma r^{2}\left(1-\frac{2 r}{3 R}+\frac{R \Delta P}{3 \gamma}\right)
$$

where $\gamma$ is the surface tension of the liquid-gas interface, $R$ is the initial radius of the bubble for which $P_{\text {eq }}$ is obtained, $r$ is the actual radius of the bubble, and $\Delta P=P-P_{\text {eq }}$.

For $\Delta P=0$, the bubble of radius $R$ is in an unstable equilibrium state, for which $\mathcal{F}$ is maximum, as shown in Fig. 2. If $\Delta P$ is increased to a positive value, the $\mathcal{F}$ curve shifts to the right, and the bubble is no longer at equilibrium. The system will decrease its free energy by dissolving the gas into the water, thus reducing the bubble radius. Conversely, if $\Delta P$ is decreased to a negative value, the opposite transfer will take place and the bubble will swell. The rate of growth or shrinking depends on the departure from the equilibrium pressure $\Delta P=P-P_{\text {eq }}$.

In our experiments, the temperature in the device is kept constant, while the pressure in the observation chamber is varied using a pressure controller. In this way, we can force the air in the bubble to dissolve into the surrounding water or vice versa. This control of the bubble size through pressure control closely mimics the exchange that takes place because of an overpressure or underpressure experienced by each bubble in a foam $[31,32]$.

The pair $\left(R, P_{\text {eq }}\right)$ must be tuned together before the armor strength can be determined. A typical experiment therefore begins by shrinking the bubble to a radius for which the particles at the interface are nearly jammed since the bubble surface is initially only partially covered. This is done by increasing $P$ well above a rough estimate of $P_{\text {eq }}$ (typically up to $100 \mathrm{mbar}$ ). Once this radius $R$ is reached, $P$ is finely tuned until the bubble size remains stationary for several minutes, indicating that the equilibrium pressure $P_{\text {eq }}$ has been found for the current bubble with a nearly jammed shell.

\section{Measurement of armor resistance}

When the bubble is armored, the particles can resist the dissolution for pressures up to a limiting overpressure that we wish to determine. For this, $\Delta P$ is increased stepwise, from a few millibars above $P_{\text {eq }}$ until buckling is observed, as summarized in Fig. 3(a). Between each of these steps, a short period of negative $\Delta P$ allows the bubble to swell again before the next pressure increase, in order to release any stresses that build up during the dissolution step.

The evolution of the armored bubble is monitored over time (see movie 2 in Ref. [26]), and the projected area of the armor is extracted by image analysis as shown in Fig. 3(a). The plot shows a first range of pressures for which the area is stable over a period of two hours, indicating that the armor is capable of blocking the dissolution of the gas into the surrounding medium at these values $(\Delta P \in[6-18] \mathrm{mbar}$ in this example). The shell remains spherical and smooth in this regime, as shown in the insets of Fig. 3(a) and in movie 2 of Ref. [26]. When the bubble inflates during the periods of negative $\Delta P$, a crack appears in the armor and then closes again as $\Delta P$ returns to a positive value. This crack can take different orientations and differs strongly from the initial condition for the experiment where the particles were loosely packed (see Fig. 1 of Ref. [26]).

At a particular value of $\Delta P$ (denoted $\Delta P_{\text {collapse }}$ ), the measured area sharply decreases with time, indicating the sudden buckling of the colloidal shell. Above this value, the armor is no longer stable and the bubble dissolves completely into the water, leaving the colloidal particles in suspension. The same behavior is observed for all of the particle sizes that we have tested, as shown, for instance, in Fig. 3 and in Ref. [26] (Fig. 1) for larger particles.

$\Delta P_{\text {collapse }}$ characterizes the resistance of each armored bubble against gas dissolution. Its variation with the physical parameters is determined by repeating the experiment for several armor radii and three different particle sizes. The results are presented in Fig. 3(b), where $\Delta P_{\text {collapse }}$ is plotted against $1 / R$, the inverse of the radius of the spherical armor in the jammed state before buckling occurs. We observe that $\Delta P_{\text {collapse }}$ decreases when the size of the shell increases. Indeed, a linear fit between $\Delta P_{\text {collapse }}$ and $1 / R$ shows good agreement with the data up to $1 / R \simeq$ $0.03 \mu \mathrm{m}^{-1}(R \simeq 33 \mu \mathrm{m})$, as shown by the dashed line in Fig. 3(b). The slope of the fitted line is $104 \mathrm{mN} / \mathrm{m}$, which corresponds to $1.5 \gamma$, where $\gamma=70 \mathrm{mN} / \mathrm{m}$ is the estimated surface tension. Surprisingly, $\Delta P_{\text {collapse }}$ is nearly independent of the diameter of the particles that make up the armor: A variation of a factor 9 in particle diameter only leads to a minor variation in the measured collapse pressures. 

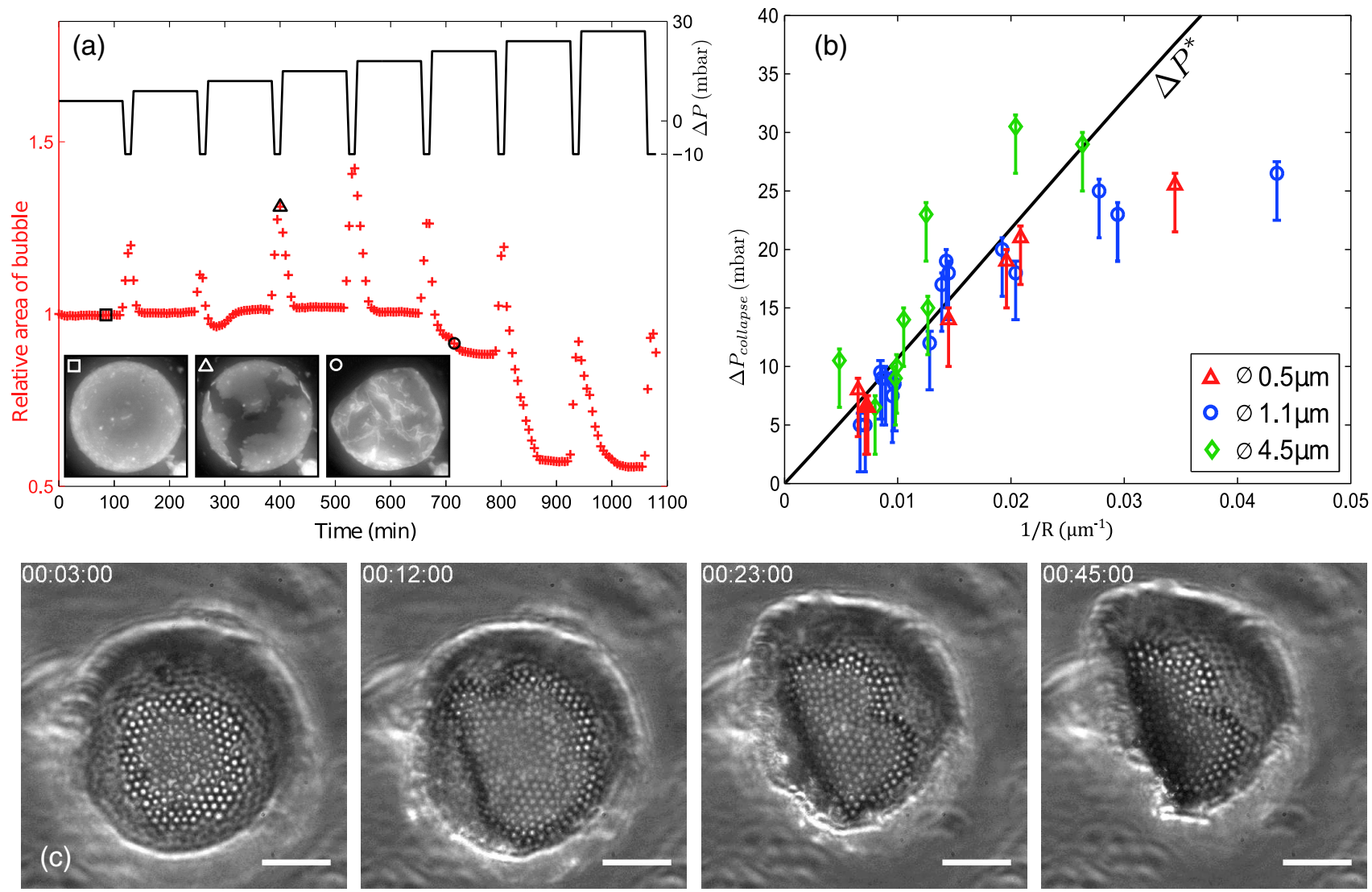

FIG. 3. (a) Typical evolution of the bubble projected area (plus) with the imposed overpressure $\Delta P$ (minus) over time. The insets are fluorescence micrographs from the corresponding experiment. The figure shows the blocked armor (square, $R=100 \mu \mathrm{m}$ ), the appearance of fractures during the bubble swelling step (triangle), and finally the buckled armor (circle). (b) Plot of $\Delta P_{\text {collapse }}$ as a function of $1 / R$, for three particle diameters. The error bars are estimated from the uncertainty in the measurement of $P_{\text {eq }}$ and the size of the $\Delta P$ steps. The black line shows the value of collapse pressure predicted by our model $\Delta P^{*} \approx 1.57 \gamma / R$. (c) Detail of the buckling event for a bubble armored with $4.5 \mu \mathrm{m}$ beads dissolving at $\Delta P=50$ mbar. Scale bars are $30 \mu \mathrm{m}$.

This failure mechanism is catastrophic in the sense that once the threshold is reached, the buckled region grows until no gas is left inside the bubble, even for a fixed $\Delta P$ [Fig. 3(c) and movie 3 in Ref. [26]]. However, if $\Delta P$ is decreased again to a negative value, the armor can reswell and regain its mechanical rigidity in a nonbuckled spherical state. This reversibility suggests that the liquid-gas contact line on the particles is pinned on the solid surface, which is confirmed by the fact that the particles never desorb from the interface: They stay attached together as a continuous granular material until the bubble has completely disappeared. These observations suggest that the air-water interface acts like a spring that pulls the armor back into place if the stress is released.

\section{MODELING THE ARMOR BEHAVIOR}

We now seek a model to reproduce the threshold value of $\Delta P$ at the onset of collapse of the colloidal shell, as well as the buckling mode. To do so, we consider a spherical shell of radius $R$ that undergoes an infinitesimal isotropic compression (radius variation $\delta R$ ). This allows us to calculate the energy variation $\left(W_{\text {diss }}\right)$ due to the gas dissolution in the liquid and compare it to the energetic cost of buckling the shell $\left(W_{\text {shel1 }}\right)$, as detailed below.

The dissolution cost is estimated from Eq. (1) and by noting that the volume variation associated with the shell compression is $\delta V=4 \pi R^{2} \delta R$. The energy $W_{\text {diss }}$ can therefore be estimated, per unit surface area of the shell, as

$$
W_{\text {diss }}=-\left.\frac{\delta V}{4 \pi R^{2}} \frac{\partial \mathcal{F}}{\partial V}\right|_{r=R}=-\Delta P \delta R
$$

This result is equivalent to saying that the shell is submitted to an external overpressure $\Delta P$, whose work $W_{\text {diss }}$ is done over the compression $\delta R$. Note that $\Delta P$ here is the departure from the equilibrium pressure $\left(P-P_{\text {eq }}\right)$ and that this result does not require any knowledge of the pressure within the bubble.

In order to estimate the energetic cost of deformation of the shell $W_{\text {shell }}$ per unit surface, we reduce the problem to the more tractable study of a 1D fiber [see Fig. 4(a)]. This fiber is an arbitrary line of $N$ rigid beads of diameter $a$ taken 

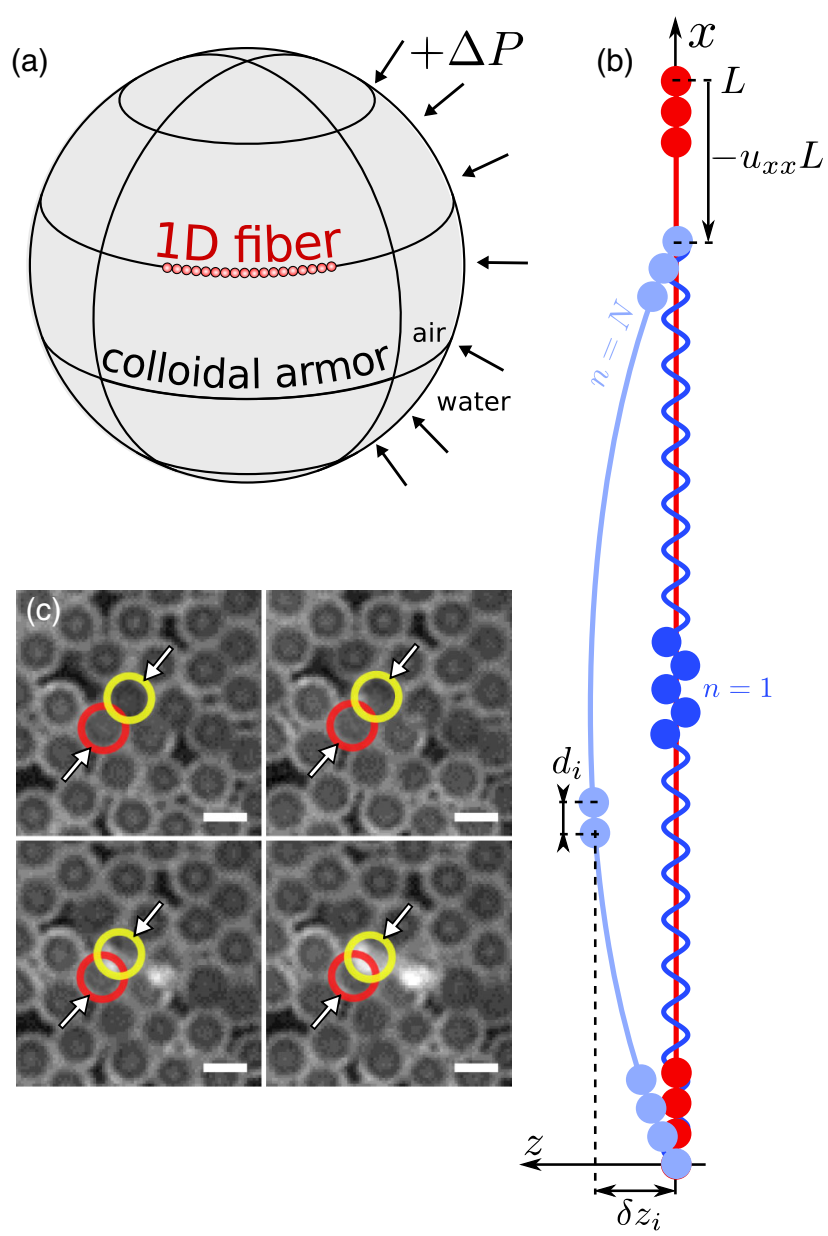

FIG. 4. (a) Sketch of the colloidal armor with an arbitrary 1D fiber highlighted in red. (b) Sketch of the buckling of the fiber. The red line shows the undeformed initial state of the fiber of length $L$; the blue lines are the deflected sinusoidal states. Light blue is the lowest frequency mode; dark blue is the highest frequency mode with alternating beads. (c) Detail from fluorescence micrographs of the buckling event (movie 4 in Ref. [26]), where the beads overlaid in red and yellow overlap and come out of the plane of the monolayer. The direction of the compression is indicated by the white arrows. Scale bars are $5 \mu \mathrm{m}$.

on the shell surface, having a total length $L$ and width $a$. These beads are adsorbed on the liquid-gas interface (surface tension $\gamma$ ), resulting in a radial restoring force that tends to hold the beads at an equilibrium position, defined here as $z=0$ [Fig. 4(b)]. The expression of the energy associated with this capillary restoring force has been derived previously. Indeed, the energy of a single bead trapped on a fluid-fluid interface minus the energy of the same bead completely immersed in the liquid is [33]

$$
\Delta E=\pi a z\left(\gamma_{s v}-\gamma_{s l}-\gamma_{l v}\right)+\pi z^{2} \gamma_{l v},
$$

where $z$ is the depth of penetration of the bead into the fluid interface. The equilibrium position for which $\Delta E$ is minimum is attained for $z_{0}=(1-\cos \theta) a / 2$ (using the
Young-Dupré relation), and the value of the minimum is $\Delta E_{\min }=-\pi \gamma z_{0}^{2}$. The energy variation $\Delta E-\Delta E_{\min }=w_{\gamma}^{i}$, which is the capillary potential energy for each bead $i$ for a vertical displacement $z-z_{0}=\delta z_{i}$, can then be written as

$$
w_{\gamma}^{i}=\pi \gamma \delta z_{i}^{2} .
$$

The compression of the armor radius leads to a longitudinal compression of the fiber, which results in a uniaxial strain $u_{x x}$. Since the beads are rigid, they must move out of the plane of the shell to comply with the $x$-axis displacement. A sinusoidal profile of amplitude $A$ is assumed to describe the out-of-plane displacements [see Fig. 4(b)]:

$$
\delta z_{i}=A \cos \left(\frac{\pi i}{n}\right) \quad \text { with } \quad n \in \mathbb{N}, \quad n \in[1, N] .
$$

We can then calculate the longitudinal ( $x$-axis) distance $d_{i}$ between two adjacent beads $i$ and $i+1$ at different deflections ( $z$ axis), for infinitesimal displacements $A \ll a$. One can then express the strain as (see Appendix B for details)

$$
u_{x x}=\frac{\sum_{i=1}^{n} d_{i}-a n}{a n}=-\frac{A^{2}}{2 a^{2}}\left[1-\cos \left(\frac{\pi}{n}\right)\right] .
$$

The total energy cost per unit surface area $W_{\text {shell }}$ of the deformed shell can now be estimated by summing the energies necessary to displace each bead over one period of the sinusoidal deflection $\delta z_{i}$. Using Eqs. (4), (5), and (6), and noting that the uniaxial strain $u_{x x}$ in any fiber of the shell is $u_{x x}=\delta R / R$, we have

$$
W_{\text {shel1 }}=\sum_{i=1, n} \frac{w_{\gamma}^{i}}{n \times a^{2}}=-\frac{\pi \gamma \delta R}{R\left[1-\cos \left(\frac{\pi}{n}\right)\right]} .
$$

Now, by equating $W_{\text {shell }}$ and $W_{\text {diss }}$, the value of the threshold pressure above which mechanical stability is lost can be obtained as

$$
\Delta P_{n}^{*}=\frac{\pi \gamma}{R\left[1-\cos \left(\frac{\pi}{n}\right)\right]} .
$$

This equation predicts different threshold pressures depending on the buckling mode $n$, with the lowest threshold $\Delta P^{*}$ being attained for $n=1: \Delta P_{1}^{*}=\pi \gamma /(2 R) \simeq 1.57 \gamma / R$, which corresponds to the smallest possible wavelength.

This prediction reproduces the three major observations in our experiments: first, the scaling of $\Delta P^{*}$ with the bubble radius as $1 / R$. Second, its insensitivity to the particle size agrees with the quantitative measurements shown in Fig. 3(b). Third, the most unstable mode is predicted to be $n=1$, which corresponds to the highest available spatial frequency with alternating beads [Fig. 4(b)]. Experimentally, this translates to the emergence of localized dislocations 
between adjacent beads at the onset of the instability. Once such a dislocation appears, the shell is expected to lose its mechanical stability just like an arch. This is indeed what is observed in the experiments [see Fig. 4(c) and movie 4 in Ref. [26]), where the buckling takes place through localized events that lead to total collapse.

This behavior is fundamentally different from what would be obtained with an elastic hollow shell [34]. In the continuous elastic case, the deformation energy cost would be proportional to the squared mean curvature of the deflection, rather than the squared amplitude as for the rigid particles. This would lead to $W_{\text {shell }}^{\text {elast }} \propto \mathrm{u}_{x x} / n^{2}$, which leads to a least-energetic cost when $n$ is maximum, that is to say, for large wavelength deformation, as in the classical Euler buckling. Moreover, the critical pressure would scale with $1 / R^{2}$ [34], also contrasting with our measurements. Note, however, that our results and analysis agree with previously observed behavior of armored interfaces, as described in Appendix $\mathrm{C}$ below.

\section{GENERALIZATION TO A FOAM}

The results above focused on the evolution of an isolated armored bubble, whose thermodynamic parameters were controlled externally. However, the final goal is to understand evolution of a foam, which is made up of a large number of interacting bubbles. Although the number of different scenarios can be large, we can readily apply our results and model above to the case where the bubbles in the foam remain spherical. This condition is verified when the foam is wet, i.e., with a liquid fraction above $30 \%$. This range of liquid fraction is a relevant regime for a wide variety of industrial applications: For instance, it is the case in many food products [35], such as ice cream [36], but also for metallic [37] or polymeric [38] foams, or cellular concrete [39]. In such foams, the spherical bubbles experience a dissolution pressure $\Delta P_{\text {foam }}$ that depends on their own size, compared to an effective size representing the surrounding foam. Lemlich derived a model based on a mean-field approximation to estimate this pressure from the statistical distribution of bubble sizes in the foam [31,32]. He proposed a model that treated the foam as an infinite liquid medium in equilibrium with a fictitious bubble of radius $R_{\text {Lemlich. }}$.

This radius $R_{\text {Lemlich }}$ is estimated by assuming a Fick's law for the gas exchange between the bubbles, where the mass flux is proportional to the pressure difference between the bubbles and the liquid. By mass conservation, the total flux summed over every bubble should be zero. This yields an expression of $R_{\text {Lemlich }}$ as a function of the probability density function of the bubble-size distribution in the foam, $F_{l}(r)$, as

$$
R_{\text {Lemlich }}=\frac{\int_{r=0}^{+\infty} r^{2} F_{l}(r) d r}{\int_{r=0}^{+\infty} r F_{l}(r) d r} .
$$

Therefore, in a foam described by Lemlich's model, each bubble experiences the dissolution pressure

$$
\Delta P_{\text {foam }}=2 \gamma\left(\frac{1}{R}-\frac{1}{R_{\text {Lemlich }}}\right)
$$

However, for a single bubble of radius $R$ immersed in water in our microfluidic device, $R_{\mathrm{eq}}(P)$ is the radius of the bubble that would be in equilibrium with the liquid for a given pressure $P$, temperature, and dissolved gas concentration. Using the expression of $R_{\text {eq }}$ Eq. (B1) (see Appendix B) and Henry's law, we obtain

$$
\Delta P=2 \gamma\left(\frac{1}{R}-\frac{1}{R_{\mathrm{eq}}(P)}\right),
$$

which is the dissolution pressure felt by the bubble of radius $R$ in our microfluidic chamber. This is equivalent to the pressure felt by a bubble in a foam where we would have $R_{\text {eq }} \equiv R_{\text {Lemlich }}$. This shows that our device simulates the environment of a foam, with $\Delta P \equiv \Delta P_{\text {foam }}$.

The behavior of any armored bubble in a foam can therefore be predicted by supposing a size distribution in the foam and comparing the resulting pressure that acts on each of these bubbles with our prediction of $\Delta P^{*}$. We perform this analysis by studying a log-normal distribution of bubble sizes, of constant mean but variable standard deviation. The overpressure or underpressure experienced by each bubble depend on its own size and on the size distribution in the foam, as shown by the colored hyperbolas in Fig. 5(a). These curves for $\Delta P_{\text {foam }}$ intersect the prediction of $\Delta P^{*}$ [Eq. (8)] at different locations depending on the initial polydispersity [Fig. 5(a), inset], thus defining a cutoff radius $R_{\text {cut }}$ that distinguishes stable from unstable regimes: Bubbles whose radius is above $R_{\text {cut }}$ are stabilized by their armor, while those whose radius is below $R_{\text {cut }}$ are expected to buckle.

It is more informative to calculate the number fraction of collapsed bubbles in the foam (dissolved fraction) by summing the number of bubbles below $R_{\text {cut }}$. The model for foams with no particles at the interfaces predicts that over $50 \%$ of the bubbles will start to dissolve at the beginning of the foam aging, even for very monodisperse initial distributions, as shown in Fig. 5(b).

In contrast, when the bubbles are armored, less than $0.1 \%$ of them are predicted to collapse and dissolve, even for polydispersity up to $40 \%$. In that case, a very small volume of gas is transferred from those $0.1 \%$ to the rest of the foam, meaning that the global bubble-size distribution is essentially unaltered. Consequently, the particle coverage of the bubbles remains unchanged, and no topological rearrangements are expected to occur. A foam meeting this polydispersity condition would thus be stable against Ostwald ripening from the very beginning of its aging. 

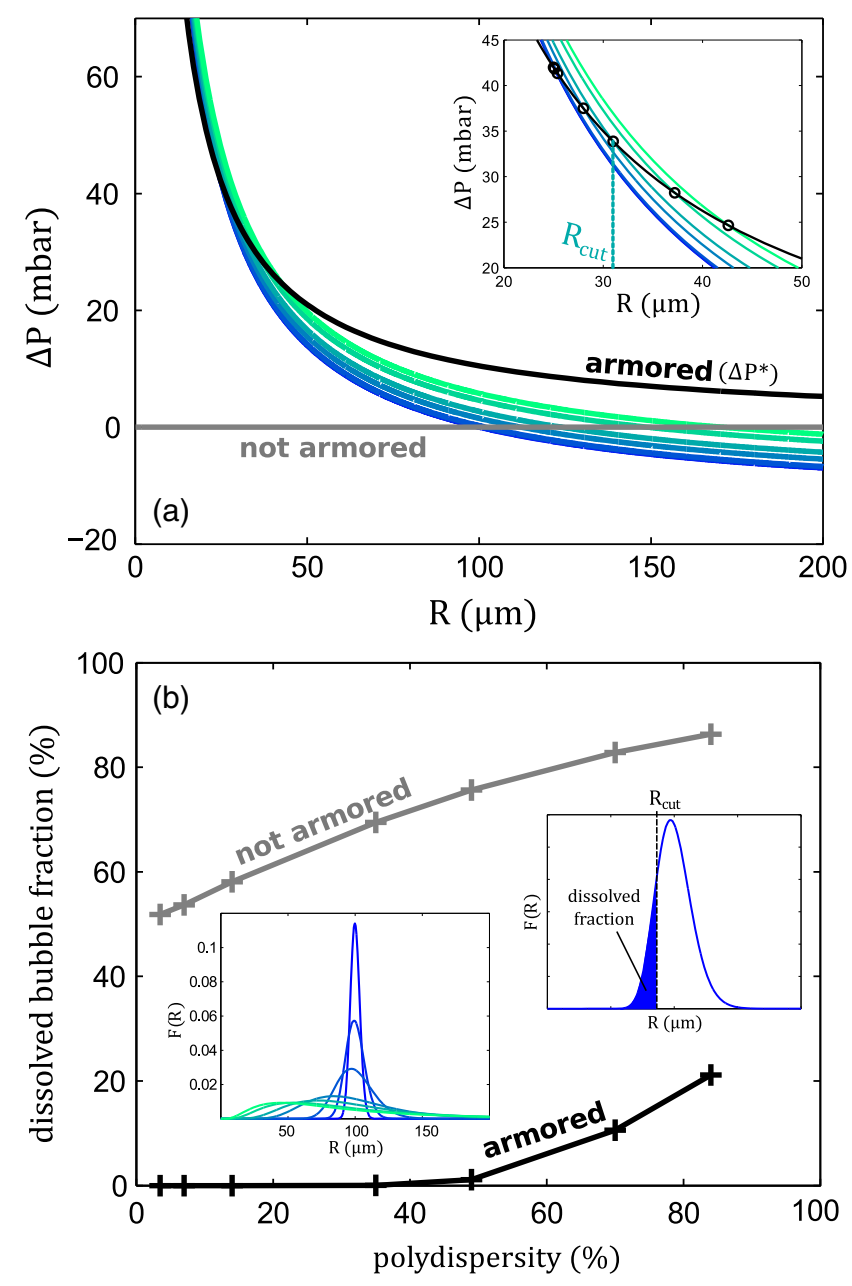

FIG. 5. (a) The blue-green curves display the overpressure felt by a bubble as a function of its inverse radius $R$ in a foam with a log-normal bubble-size distribution. The mean of the distribution is kept constant at $100 \mu \mathrm{m}$, while the standard deviation is varied from $3.5 \mu \mathrm{m}$ (blue) to $84 \mu \mathrm{m}$ (green), as shown in the lower inset. The black line represents the resistance $\Delta P^{*}$ of a colloidal shell as defined by our model and our experiments. Bubbles subjected to a pressure below this critical value will collapse and disappear, while the others will remain stable. The case of unarmored bubbles $(\Delta P=0)$ is displayed in gray for comparison. The inset is a close-up of the region where $\Delta P=\Delta P^{*}$ (०), which defines the cutoff radii $R_{\text {cut }}$. (b) The percentage of bubbles that collapse or dissolve under the influence of the whole foam, in the armored case (black) and the unarmored case (gray). The polydispersity is calculated as the standard deviation of the distribution divided by the mean. The buckled percentage is calculated by integrating the probability density function on $\left[0, R_{\text {cut }}\right]$, as shown in the right inset. The left inset shows the probability density functions $F(R)$ used for the calculations, with increasing polydispersity.

\section{DISCUSSION}

As microfluidic tools continue to develop and to gain in robustness, they provide qualitatively new methods to address questions in different areas of science. The study shown here, which relies on the ability to generate and coat a single microscopic bubble and observe it over long periods, provides a unique approach for measuring the mechanical stability of a microscopic armor. Our results on armored bubbles provide a multiscale framework for understanding foam stabilization: The quantitative measurements of the armor strength are accounted for by a theoretical model, which is based on the energy balance between the energy gain of gas dissolution and the cost of displacing individual particles across the interface. These observations show that the stability of the armored bubbles is similar to that of an arch, emerging from the geometric arrangement of the individual bricks. As such, dislocations of individual particles lead to the shell collapse.

One consequence of this microscopic behavior is that the shell resistance departs strongly from what is expected from elasticity, contrary to what is commonly assumed $[16,40,41]$. This suggests ways to reinforce the colloidal armor by inhibiting the short-wavelength dislocations, for example, by using nonspherical or rough particles to prevent rolling or by using adhesive particles to sinter the shell. A second surprising consequence is that the effect of particle size on the stabilization is very weak, with submicron particles displaying equivalent stability as particles 10 times larger. This motivates the choice of smaller particles for foam stabilization since the material usage is reduced, and smaller colloids adsorb more easily on interfaces [5].

Finally, the model of stability on the bubble scale can be used to predict the long-term evolution of a foam by Ostwald ripening. This is suitable since the dissolution pressure applied in our experiments is a direct analog for Lemlich's model for the behavior of an individual bubble in a foam. We find that in an aging particle-stabilized foam, small bubbles will buckle before big bubbles since the dissolution pressure they experience grows faster than their resistance to it. Also, we show that the stability of the final foam is conditioned by the initial polydispersity of the bubbles, which is determined by the foaming method. This criterion can therefore be used in the choice of foaming methods for practical industrial processes.

Both the theoretical model and the microfluidic setup can naturally be generalized to physicochemical systems with more industrial relevance, including elastic shells or particles having different chemical, electrostatic, or mechanical interactions. Finally, the new physical mechanisms evidenced for particle-coated interfaces hold for liquidliquid interfaces. Our results can therefore be generalized to investigate the stability of armored droplets and particlestabilized emulsions.

\section{ACKNOWLEDGMENTS}

The authors thank Caroline Frot for help with the microfabrication. Useful discussions with Arezki Boudaoud and Emmanuel de Langre are gratefully acknowledged. 
The research leading to these results received funding from the European Research Council (ERC) Grant Agreement No. 278248 Multicell.

\section{APPENDIX A: MATERIALS AND METHODS}

\section{Microfluidics}

The experiments were conducted in PDMS (Sylgard 184, Dow Corning) microchannels, plasma bonded to glass. A mold (see Fig. 6) was first micromilled in a brass block using a CNC micromilling machine (Minitech), following a 3D model of the channel drawn using CAM software (Rhinoceros). Liquid PDMS was then cast over the mold and cured on a hot plate at $150{ }^{\circ} \mathrm{C}$ for at least $24 \mathrm{~h}$. This thermal aging was necessary to reduce the hydrophobic recovery of the PDMS after plasma treatment [42]. The patterned PDMS was then sealed to a glass slide after $30 \mathrm{~s}$ in an air plasma, and the resulting channel was immediately filled with distilled water (Milli-Q) to ensure that the walls remain hydrophilic. The microfluidic chips were stored in a $100 \%$ humidity sealed container in order to prevent drying and to keep the channels hydrophilic. Fluids were flowed into the channels using Teflon (PTFE) tubing and glass syringes driven by syringe pumps (Nemesys, Cetoni). The ambient pressure inside the microsystem was controlled by an external pressure controller (MFCS, Fluigent). The temperature was regulated at $50{ }^{\circ} \mathrm{C}$ by placing the microchip on a heated microscope stage (Warner Instruments). The bubbles were monitored by phase contrast or fluorescence microscopy (Eclipse Ti, Nikon) using a CCD camera (Insight, SPOT). We estimated the evolution of the volume of gas inside the bubble by measuring the projected area of the bubble by gray-level threshold image analysis (Matlab).

\section{Particles}

The particles used for the experiments were yellowgreen fluorescent polystyrene (PS) microbeads, charge stabilized by covalently bonded carboxylate groups.

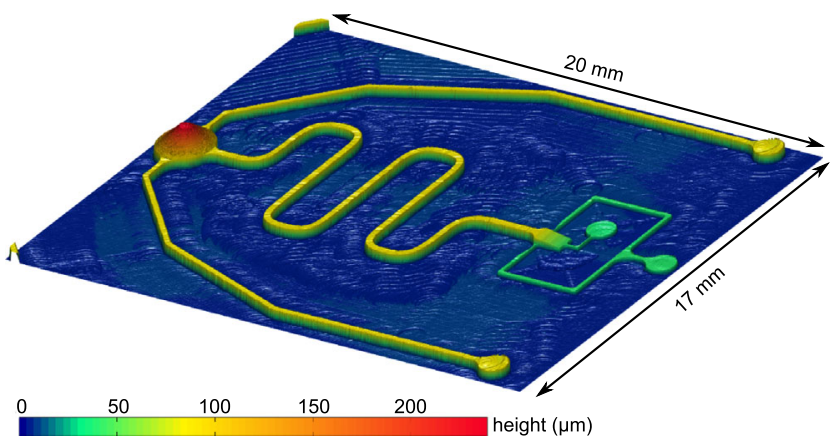

FIG. 6. Three-dimensional view of the brass mold used for the casting of the microchips. It was obtained with an optical profilometer (Zygo) by stitching images from multiple acquisitions. The height of the coating channel is $80 \mu \mathrm{m}$, and its width is $300 \mu \mathrm{m}$. The slope of the bubble injector is $4 \%$.
They were washed three times with distilled water by centrifugation-sonication cycles and finally redispersed in DI water with $0.5 \mathrm{M}$ sodium chloride $(\mathrm{pH}=7)$ to a final solid concentration of $0.5 \% \mathrm{w} / \mathrm{w}$. Salty water was used in order to screen the electrostatic repulsion between the particles and the interface [5], to facilitate adsorption. In our experiments, we used three different particle diameters: 0.5, 1.1 (Fluospheres, Life Technologies), and $4.5 \mu \mathrm{m}$ (Fluoresbrite, Polysciences).

\section{APPENDIX B: DETAILED CALCULATIONS}

\section{Calculation of the free energy of the dissolving bubble}

Let us consider a gas bubble of radius $R$ in an infinite liquid medium at a given thermodynamic state: temperature $T$, pressure $P$, liquid-gas surface tension $\gamma$, liquid-vapor pressure $P_{\infty}$, dissolved gas concentration $C$, and saturation concentration of gas in the liquid through a flat interface $C_{\text {sat }}(P, T)$. A bubble is in unstable equilibrium if it has a radius $R_{\mathrm{eq}}(P)$, the so-called Gibbs critical radius, for which the free energy $\mathcal{F}$ of the system is maximum. $R_{\text {eq }}$ can be expressed as [30]

$$
\frac{1}{R_{\mathrm{eq}}(P)}=\frac{1}{2 \gamma}\left[P_{\infty}+P\left(\frac{C}{C_{\mathrm{sat}}(P)}-1\right)\right] \text {. }
$$

Conversely, we can find a pressure value $P_{\text {eq }}$ for which the initial state matches an unstable equilibrium state, that is, $R_{\text {eq }}\left(P_{\text {eq }}\right)=R$. Ward et al. [30] derived an approximate expression of the free energy $\mathcal{F}$ for a bubble of radius $r$ in the vicinity of $R_{\text {eq }}$ :

$$
\mathcal{F}=4 \pi \gamma\left(r^{2}-\frac{2}{3} \frac{r^{3}}{R_{\mathrm{eq}}}\right) .
$$

Bubbles with radius $r>R_{\text {eq }}$ will swell, while those with $r<R_{\text {eq }}$, will shrink. The concentration of gas being considered low, it is reasonable to assume that it follows Henry's law, $C_{\text {sat }}(P)=K_{H} P$, where $K_{H}$ is the Henry constant of the air dissolved in the water at temperature $T$. Inserting the expression of $C_{\text {sat }}$ into Eq. (B1) written for $R_{\text {eq }}\left(P_{\text {eq }}\right)=R$ yields an expression of $C$, which remains constant throughout the experiment. Then, using this in Eq. (B1) and combining with Eq. (B2) gives the result in Eq. (1).

We verify experimentally the ability to control the evolution of an air-in-water bubble by varying the pressure around it. Figure 7 shows the time evolution of the relative radius of the bubble for different values of $\Delta P$, for a typical experimental temperature of $50{ }^{\circ} \mathrm{C}$ and an initial bubble radius of $R=120 \mu \mathrm{m}$. In these conditions, the equilibrium pressure is $P_{\mathrm{eq}}=80 \mathrm{mbar}$. At that pressure value, corresponding to $\Delta P=0$, the radius of the bubble changes by less than $1 \%$ over 20 minutes, which indicates that the 
unstable state of equilibrium has been reached. As soon as the value of $\Delta P$ is increased (resp. decreased), the radius of the bubble decreases (resp. increases). It can also be noticed that the rate of variation of the bubble radius is related to the absolute value of $\Delta P$. The further the system is driven out of equilibrium, the higher the rate of variation of $r$.

\section{Calculation of the strain of the armor fiber}

Using the Pythagorean theorem and assuming $a \ll A$,

$$
\begin{aligned}
d_{i} & =\sqrt{a^{2}-A^{2}\left[\cos \left(\frac{\pi i}{n}\right)-\cos \left(\frac{\pi(i+1)}{n}\right)\right]^{2}} \\
& \simeq a\left(1-\frac{A^{2}}{2 a^{2}}\left[\cos \left(\frac{\pi i}{n}\right)-\cos \left(\frac{\pi(i+1)}{n}\right)\right]^{2}\right) .
\end{aligned}
$$

The length of one period is then

$$
\begin{aligned}
\sum_{i=1}^{n} d_{i} & =a n-\frac{A^{2}}{2 a} \sum_{i=1}^{n}\left[\cos \left(\frac{\pi i}{n}\right)-\cos \left(\frac{\pi(i+1)}{n}\right)\right]^{2} \\
& =a n-\frac{n A^{2}}{2 a}\left[1-\cos \left(\frac{\pi}{n}\right)\right] .
\end{aligned}
$$

\section{APPENDIX C: COMPARISON WITH PREVIOUS RESULTS}

The mechanical resistance of armored interfaces has been measured by other authors, particularly in Ref. [13] (water-in-oil droplets) and Ref. [17] (hemispherical waterin-air droplets). As summarized in Table I, our study covers a range or particle-to-bubble diameters that bridges these two studies while overlapping slightly with them. In spite of the differences in experimental setups, our measurements and theoretical predictions for the collapse pressure quantitatively agree with the results presented in these studies, as described below.

Monteux et al. [13] investigated the deflation of a particle-coated millimetric droplet of decane in water. Despite observing jamming of the particles at the interface and a subsequent collapse of the shell, they did not address the question of the mechanical resistance of such armor. Indeed, they controlled the volume of the droplet rather than the pressure, which would make a measurement of the collapse pressure tricky. Their most relevant measurements

TABLE I. Range of particle diameter to bubble radius ratios $(a / R)$ covered by the three studies.

\begin{tabular}{lccc}
\hline \hline Reference & $\operatorname{Min}(a / R)$ & $\operatorname{Max}(a / R)$ & Max/Min \\
\hline Monteux et al. $[13]$ & $2.1 \times 10^{-4}$ & $2.8 \times 10^{-3}$ & 13 \\
Pitois et al. $[17]$ & $2.0 \times 10^{-2}$ & $1.8 \times 10^{-1}$ & 9 \\
Current study & $1.6 \times 10^{-3}$ & $5.9 \times 10^{-2}$ & 36 \\
\hline \hline
\end{tabular}

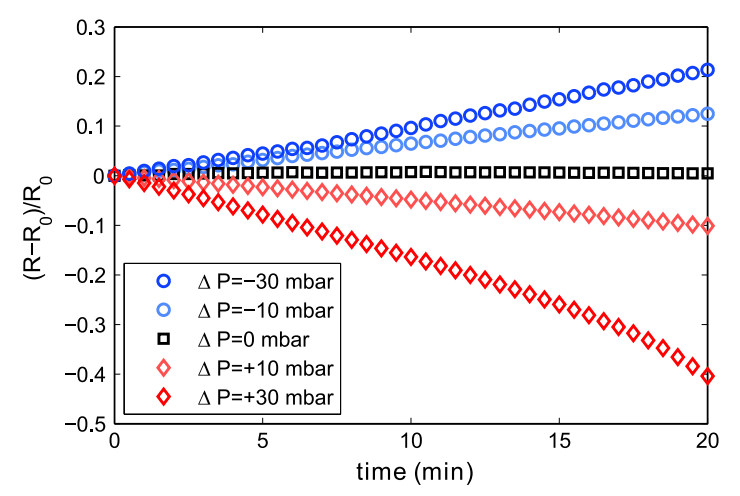

FIG. 7. Measurement of the time evolution of the normalized radius of an air bubble in water, for different values of $\Delta P$.

were performed in a flat Langmuir trough, where they found a buckling surface pressure $\Pi$ that is independent of the particle size (regime III at large compression values, in their Fig. 8).

This surface pressure is related to our collapse pressure since the surface pressure in an isotropically compressed spherical shell is related to the external overpressure by $\Pi=R \Delta P / 2$ (expression used in Ref. [17], p. 5). Thus, our expression $\Delta P \approx 1.57 \gamma / R$ can be translated in terms of surface pressure (using $\gamma=50.3 \mathrm{mN} / \mathrm{m}$ for the decane/water interface): $\Pi \approx 0.78 \gamma=0.78 \times 50.3 \mathrm{mN} / \mathrm{m}=$ $39.5 \mathrm{mN} / \mathrm{m}$. This value is in very good agreement with data of Monteux et al., who find a value around $40 \mathrm{mN} / \mathrm{m}$, with no observable dependence on particle size.

In the study by Pitois et al. [17], the authors decrease the internal pressure of an armored hemispherical droplet until they observe buckling. Their measurements of collapse pressure for two particle diameters, as reported in their Fig. 3, collapse on a single master curve when plotted as a

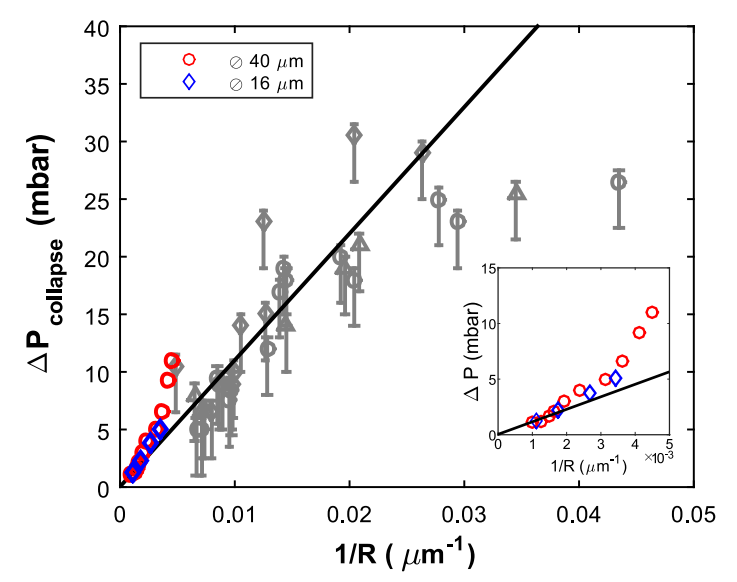

FIG. 8. Armored bubble collapse pressure as a function of the inverse radius. The black line is our model prediction, the gray symbols correspond to our experimental points, and the colored symbols correspond to measurements from Ref. [17] for two different particle diameters: $16 \mu \mathrm{m}$ and $40 \mu \mathrm{m}$. The inset is a close-up for small values of $1 / R$. 
function of the bubble radius (Fig. 8 inset). This indicates that there is no dependence on the particle diameter in their measurements. Indeed, their data align well with ours and with our theoretical prediction of the collapse pressure (Fig. 8), except for the largest values of $a / R$ for which different stabilization mechanisms are expected $[17,20]$.

[1] S. Cohen-Addad, R. Höhler, and O. Pitois, Flow in Foams and Flowing Foams, Annu. Rev. Fluid Mech. 45, 241 (2013).

[2] J. Pierre, B. Dollet, and V. Leroy, Resonant Acoustic Propagation and Negative Density in Liquid Foams, Phys. Rev. Lett. 112, 148307 (2014).

[3] M. Hashimoto, B. Mayers, P. Garstecki, and G. M. Whitesides, Flowing Lattices of Bubbles as Tunable, SelfAssembled Diffraction Gratings, Small 2, 1292 (2006).

[4] I. Cantat, S. Cohen-Addad, F. Elias, F. Graner, R. Höhler, O. Pitois, F. Rouyer, and A. Saint-Jalmes, Foams: Structure and Dynamics (Oxford University, Oxford, 2013).

[5] S. Tcholakova, N. D. Denkov, and A. Lips, Comparison of Solid Particles, Globular Proteins and Surfactants as Emulsifiers, Phys. Chem. Chem. Phys. 10, 1608 (2008).

[6] W. Ramsden, Separation of Solids in the Surface-Layer of Solutions and Suspensions, Proc. R. Soc. London 72, 156 (1903).

[7] B. P. Binks and R. Murakami, Phase Inversion of ParticleStabilized Materials from Foams to Dry Water, Nat. Mater. 5, 865 (2006).

[8] U. T. Gonzenbach, A. R. Studart, E. Tervoort, and L. J. Gauckler, Ultrastable Particle-Stabilized Foams, Angew. Chem. 45, 3526 (2006).

[9] S. Lam, E. Blanco, S. K. Smoukov, K. P. Velikov, and O. D. Velev, Magnetically Responsive Pickering Foams, J. Am. Chem. Soc. 133, 13856 (2011).

[10] A. Stocco, E. Rio, B. P. Binks, and D. Langevin, Aqueous Foams Stabilized Solely by Particles, Soft Matter 7, 1260 (2011).

[11] A. Maestro, E. Rio, W. Drenckhan, D. Langevin, and A. Salonen, Foams Stabilised by Mixtures of Nanoparticles and Oppositely Charged Surfactants: Relationship between Bubble Shrinkage and Foam Coarsening, Soft Matter 10, 6975 (2014).

[12] N. Tsapis, E. Dufresne, S. Sinha, C. Riera, J. Hutchinson, L. Mahadevan, and D. Weitz, Onset of Buckling in Drying Droplets of Colloidal Suspensions, Phys. Rev. Lett. 94, 018302 (2005).

[13] C. Monteux, J. Kirkwood, H. Xu, E. Jung, and G. G. Fuller, Determining the Mechanical Response of Particle-Laden Fluid Interfaces Using Surface Pressure Isotherms and Bulk Pressure Measurements of Droplets, Phys. Chem. Chem. Phys. 9, 6344 (2007).

[14] V. Garbin, J. C. Crocker, and K. J. Stebe, Forced Desorption of Nanoparticles from an Oil Water Interface, Langmuir $\mathbf{2 8 ,}$ 1663 (2012).

[15] B. Laborie, F. Lachaussée, E. Lorenceau, and F. Rouyer, How Coatings with Hydrophobic Particles May Change the Drying of Water Droplets: Incompressible Surface versus Porous Media Effects, Soft Matter 9, 4822 (2013).
[16] G. Lagubeau, A. Rescaglio, and F. Melo, Armoring a Droplet: Soft Jamming of a Dense Granular Interface, Phys. Rev. E 90, 030201 (2014).

[17] O. Pitois, M. Buisson, and X. Chateau, On the Collapse Pressure of Armored Bubbles and Drops, Eur. Phys. J. E 38, 48 (2015).

[18] S. S. Datta, H. C. Shum, and D. A. Weitz, Controlled Buckling and Crumpling of Nanoparticle-Coated Droplets, Langmuir 26, 18612 (2010).

[19] A. Bala Subramaniam, M. Abkarian, and H. A. Stone, Controlled Assembly of Jammed Colloidal Shells on Fluid Droplets, Nat. Mater. 4, 553 (2005).

[20] M. Abkarian, A. Bala Subramaniam, S.-H. Kim, R. Larsen, S.-M. Yang, and H. A. Stone, Dissolution Arrest and Stability of Particle-Covered Bubbles, Phys. Rev. Lett. 99, 188301 (2007).

[21] R. Dangla, S. Lee, and C. N. Baroud, Trapping Microfluidic Drops in Wells of Surface Energy, Phys. Rev. Lett. 107, 124501 (2011).

[22] R. Dangla, S. C. Kayi, and C. N. Baroud, Droplet Microfluidics Driven by Gradients of Confinement, Proc. Natl. Acad. Sci. U.S.A. 110, 853 (2013).

[23] S. Lee, F. Gallaire, and C. N. Baroud, Interface-Induced Recirculation within a Stationary Microfluidic Drop, Soft Matter 8, 10750 (2012).

[24] E. Fradet, P. Abbyad, M. H. Vos, and C. N. Baroud, Parallel Measurements of Reaction Kinetics Using UltralowVolumes, Lab Chip 13, 4326 (2013).

[25] A. Yamada, S. Lee, P. Bassereau, and C. N. Baroud, Trapping and Release of Giant Unilamellar Vesicles in Microfluidic Wells, Soft Matter 10, 5878 (2014).

[26] See Supplemental Material at http://link.aps.org/ supplemental/10.1103/PhysRevX.6.011010 for additional figure and movies.

[27] F. Zoueshtiagh, M. Baudoin, and D. Guerrin, Capillary Tube Wetting Induced by Particles: Towards Armoured Bubbles Tailoring, Soft Matter 10, 9403 (2014).

[28] A. P. Kotula and S. L. Anna, Probing Timescales for Colloidal Particle Adsorption Using Slug Bubbles in Rectangular Microchannels, Soft Matter 8, 10759 (2012).

[29] P. S. Epstein and M. S. Plesset, On the Stability of Gas Bubbles in Liquid-Gas Solutions, J. Chem. Phys. 18, 1505 (1950).

[30] C. A. Ward, A. Balakrishnan, and F. C. Hooper, On the Thermodynamics of Nucleation in Weak Gas-Liquid Solutions, J. Basic Eng. 92, 695 (1970).

[31] R. Lemlich, Prediction of Changes in Bubble Size Distribution Due to Interbubble Gas Diffusion in Foam, Ind. Eng. Chem. Fundam. 17, 89 (1978).

[32] S. Tcholakova, Z. Mitrinova, K. Golemanov, N. D. Denkov, M. Vethamuthu, and K. P. Ananthapadmanabhan, Control of Ostwald Ripening by Using Surfactants with High Surface Modulus, Langmuir 27, 14807 (2011).

[33] P. Pieranski, Two-Dimensional Interfacial Colloidal Crystals, Phys. Rev. Lett. 45, 569 (1980).

[34] A. V. Pogorelov, Bending of Surfaces and Stability of Shells (American Mathematical Society, Providence, 1988).

[35] G. M. Campbell and E. Mougeot, Creation and Characterisation of Aerated Food Products, Trends Food Sci. Tech. 10, 283 (1999). 
[36] M. D. Eisner, H. Wildmoser, and E. J. Windhab, Air Cell Microstructuring in a High Viscous Ice Cream Matrix, Colloids Surf. 263, 390 (2005).

[37] J. Banhart, Metal Foams: Production and Stability, Adv. Eng. Mater. 8, 781 (2006).

[38] J. I. González Ocampo, D. M. Escobar Sierra, and C. P. Ossa Orozco, Porous Bodies of Hydroxyapatite Produced by a Combination of the Gel-Casting and Polymer Sponge Methods, Journal of Advanced Research, doi:10.1016/ j.jare.2015.06.006 (in press).

[39] P. Petit, I. Javierre, P. H. Jézéquel, and A. L. Biance, Generation and Stability of Bubbles in a Cement
Based Slurry, Cement and Concrete Research 60, 37 (2014).

[40] D. Vella, P. Aussillous, and L. Mahadevan, Elasticity of an Interfacial Particle Raft, Europhys. Lett. 68, 212 (2004).

[41] P. A. Kralchevsky, I. B. Ivanov, K. P. Ananthapadmanabhan, and A. Lips, On the Thermodynamics of Particle-Stabilized Emulsions: Curvature Effects and Catastrophic Phase Inversion, Langmuir 21, 50 (2005).

[42] D. T. Eddington, J. P. Puccinelli, and D. J. Beebe, Thermal Aging and Reduced Hydrophobic Recovery of Polydimethylsiloxane, Sensors Actuators B 114, 170 (2006). 